Nova, N. \& Roussilhe, G. (2020). Du low-tech numérique aux numériques situés, Sciences du design, 11(1), p. 91-101.

\title{
Du low-tech numérique aux numériques situés
}

\author{
Nicolas Nova \\ Professeur associé à la Haute École d'Art et de Design \\ Chercheur associé Médialab Sciences Po Paris \\ Paris, France \\ nicolas.nova@hesge.ch
}

Gauthier Roussilhe

Designer

Pessac, France

gauthierroussilhe@protonmail.com

\section{RÉSUMÉ}

En partant de l'injonction paradoxale de concilier transition numérique et écologique, cet article décrit comment les enjeux actuels de la crise environnementale débouchent sur la notion de design numérique «low-tech ». Sur la base d'une série de projets couramment mobilisés par les acteurs du design numérique - dans des domaines aussi divers que le jeu vidéo, la création de sites web visant à minimiser les émissions de carbone, la conception de machines recyclées ou de médias dits zombies - nous proposons une critique de cette expression. Nous en tirons la notion de «numériques situés », afin de dépasser les écueils et apories de cette sobriété numérique mal définie et au fond polysémique.

MOTS CLÉS : design numérique, low-tech, crise environnementale, design de transition.

\begin{abstract}
Based on the paradoxical imperative to achieve a simultaneous digital and ecological transition, this article describes how the current environmental crises are leading to a strong interest in "lowtech" design. Drawing on a series of projects commonly discussed in design circles - ranging from video game design to websites aimed at minimizing carbon emissions, as well as recycled machines or so-called zombie media - the paper both describes and a critique of the term "lowtech" and suggest the notion of "situated digitals" in order to overcome the pitfalls of this illdefined and basically polysemic digital sobriety.
\end{abstract}

KEYWORDS : digital design, low-tech, environmental crisis, transition design. 
Nova, N. \& Roussilhe, G. (2020). Du low-tech numérique aux numériques situés, Sciences du design, 11(1), p. 91-101.

En tant que phénomène visant à renforcer l'intégration des techniques numériques dans les organisations et dans les activités du quotidien, l'injonction actuelle à la transformation numérique se heurte de fait à une crise environnementale systémique dont les symptômes et effets sont aussi multiples que bien documentés. Ce paradoxe transparaît dans la médiatisation des quantités d'énergie nécessaires pour fabriquer et alimenter nos appareils, ou faire fonctionner les centres de données (Diguet et Lopez, 2019), mais aussi du fait des problèmes liés à l'extraction de métaux ou de matériaux critiques présents dans notre instrumentarium numérique (Pitron, 2018). Pour dépasser cette opposition, de multiples propositions ont vu le jour ces dernières années. Celles-ci ont différents objectifs, comme de trouver un équilibre dans le prolongement des initiatives de développement durable par l'introduction de nouvelles technologies ou l'optimisation de processus, ou de prendre les enjeux écologiques comme un moyen de repenser la société numérique (Fing, 2019). Il peut s'agir aussi d'explorer les conditions d'une société de l'information dans un contexte d'effondrement (Tomlinson et al., 2012), voire de promouvoir une sobriété qui « passerait par des basses technologies sans doute plus rudimentaires, peut-être moins performantes, mais nettement plus économes en ressources et maîtrisables localement » (Bihouix, 2014). C'est à cette orientation dite « low-tech » que nous allons nous intéresser ici, en nous penchant sur son écho chez les designers dans le champ du numérique. Ce qualificatif semble rencontrer un certain succès chez ceux-ci, et plus largement dans les milieux intéressés à l'innovation technologique, particulièrement dans le monde francophone. Si les designers produits et les architectes ont eu leur prise de conscience des questions écologiques avec, par exemple, le recours à des matériaux biosourcés, des enjeux énergétiques ou un design dit de « transition » (Irwin, 2015), les acteurs du design numérique ne semblent que plus récemment concernés par ces enjeux. Dans ces circonstances, le low-tech est devenu une piste fertile pour cette communauté de pratiques qui s'en saisit pour faire face aux débats à propos de l'Anthropocène, ou d'un éventuel effondrement social et écologique, qui se trouve discuté lors de conférences ou de rencontres professionnelles dans le champ du design numérique ${ }^{1}$. L'essentiel des échanges évoque des interrogations ou des doutes ${ }^{2}$, mais aussi des moyens de se saisir de cette notion dans le champ des technologies de l'information et de la communication ${ }^{3}$, et de partager des recommandations pratiques (Dorne, 2019 ; Roussilhe, 2019).

Or, dans ces différents cas, les définitions de ce low-tech numérique sont globalement éludées, ou remplacées par le renvoi à des références telles que celle faite au travail de Philippe Bihouix, voire décrites par l'exemple couramment cité du Low-tech Magazine qui a développé une version de son site web alimentée par l'énergie solaire. C'est justement ce qui va nous intéresser ici. Plutôt que de prendre ce constat comme une faiblesse, nous adoptons une posture pragmatique en interrogeant cette notion à l'aune de multiples pratiques de création dans le champ du numérique. Pour cela, nous nous appuierons sur une série de cas collectés au gré de nos activités respectives de designer et d'anthropologue, lors de conférences, rencontres professionnelles, ateliers et autres échanges informels entre acteurs de la création numérique au sens large.

\footnotetext{
${ }^{1}$ Comme les conférences Blendwebmix organisées chaque automne à Lyon.

${ }^{2}$ Voir notamment les liens suivants : https://graphism.fr/du-design-a-leffondrement-par-ou-commencercollapsologie/, http://mcgodwin.com/en/talks/design-unknown-future, https://vimeo.com/342949630

${ }^{3}$ Voir par exemple cette conférence d'Alexandre Monnin et Diego Landivar à la conférence BlendWebMix https://www.youtube.com/watch?v=RXTFyA6qwDs\&list=UUVA4ZOoyUyLB LS6flBjhRg\&index=19
} 
Nova, N. \& Roussilhe, G. (2020). Du low-tech numérique aux numériques situés, Sciences du design, 11(1), p. 91-101.

\section{Ordinateurs low-tech}

Un ordinateur est un ensemble de composants électroniques extrêmement complexes qui nécessitent une large chaîne industrielle allant de l'extraction des matières premières, leur transformation, l'assemblage et l'acheminement jusqu'à la mise en décharge ou le réemploi de l'ordinateur et de ses composants. La maximisation de son usage, sa réparation, sa maintenance et son réemploi devient alors une question majeure dans un contexte de crise environnementale où l'approvisionnement en énergie et en matières premières évolue de façon critique.

Figure $1:$ Jerry Can computer

Plusieurs concepteurs ont proposé diverses approches à l'élaboration d'un ordinateur qui minimiserait l'énergie ainsi que les ressources naturelles et financières investies, et donc son impact environnemental. Une équipe de designers de l'ENSCI a par exemple conçu un ordinateur dans un bidon en plastique - objet quotidien massivement accessible dans le monde globalisé - au sein duquel des composants électroniques peu onéreux sont fixés et raccordés ${ }^{4}$. Cet ordinateur peut faire office de serveur ; il a été construit et déployé sur un grand nombre de terrains en Côte d'Ivoire ou en Algérie. L'équipe du Low-tech lab a, de son côté, documenté une façon d'assembler et d'installer un poste d'ordinateur pour moins de 30 dollars américains ${ }^{5}$ à partir du réemploi d'interfaces de seconde main et d'un minimum de composants électroniques nécessaires pour un ensemble d'usages primaires du numérique (bureautique, navigation et multimédia). Ces deux démarches sont ellesmêmes proches d'initiatives plus anciennes telles que les Zero dollar laptop workshops ${ }^{6}$ qui, dès 2007, offraient des ateliers de recyclage et de récupération d'ordinateurs et de composants informatiques ainsi que la formation de différents publics (notamment des personnes sans abri) à l'installation et à l'entretien des ordinateurs et des systèmes d'exploitation libres.

\section{Médias zombies}

Le fait de recourir au réemploi ou à des matériaux recyclés évoqués dans le cas des ordinateurs dits low-tech correspond aussi à d'autres pratiques dans le champ artistique de la musique électronique ou du new media art.

\section{Figure 2: MIDIbox SID synthesizer (Jahtari)}

Le label allemand Jahtari, spécialisé dans les hybridations singulières entre électro et reggae et publiées tant sous la forme de fichiers MP3, de disques vinyle ou de cassettes audio, construit aussi ponctuellement des instruments artisanaux. Sans volonté de production commerciale de masse, Jahtari fournit ainsi aux créateurs de musique électronique le MIDIbox SID synthesizer, un synthétiseur formé de deux modules dont le fonctionnement repose sur la récupération de microprocesseurs sonores (SID) sur de vieux ordinateurs C64 achetés sur Internet ou dans les brocantes, assemblés à des composants électroniques plus récents insérés dans une coque d'origine de cette machine. Du fait de son caractère hybride, dérivé de techniques anciennes avec ses processeurs sonores SID low-tech, cet appareil peut être considéré comme un moyen plus accessible

\footnotetext{
4 http://www.youandjerrycan.org

5 https://lowtechlab.org/wiki/Ordinateur low-tech

6 https://www.furtherfield.org/zerodollarlaptop/?page id $=2$
} 
Nova, N. \& Roussilhe, G. (2020). Du low-tech numérique aux numériques situés, Sciences du design, 11(1), p. 91-101.

de réutiliser ce type de son, et tout l'imaginaire qui lui correspond, dans une machine à l'ergonomie contemporaine (Nova, 2017).

De la même manière, la récupération de déchets technologiques et le détournement de machines obsolètes forment une logique commune à de nombreux artistes dans le champ du new media art. Benjamin Gaulon est certainement l'un des représentants les plus pertinents de cette approche, suivant la longue tradition historique de création d'art à partir de déchets et de rebuts en tout genre. Dans son projet intitulé Recycling Entertainment System, il réassemble des consoles de jeux obsolètes, transformant les restes d'une NES en une installation musicale pour six joueurs, équipée d'un contrôleur qui leur permet de jouer un instrument et, finalement, fait évoluer le jeu individuel originel en une expérience collective. Son projet ReFunct Media transforme et assemble quant à lui toute une série de dispositifs électroniques désormais dépassés en une sorte de chaîne de machines interconnectées. Contrôleurs de jeux vidéo, caméras, chaînes Hi-Fi, téléviseurs, Gameboys sont ainsi reliés entre eux pour créer une installation que le spectateur observe comme une sorte de réaction en chaîne qui produit une expérience esthétique originale en (ré)animant tous ces objets du passé.

Figure $3:$ ReFunct Media (Benjamin Gaulon)

Pour décrire ces trois exemples, auxquels on pourrait ajouter la pratique du circuit bending qui consiste à faire circuler du courant de façon imprévue dans des circuits intégrés de machines obsolètes afin de faire surgir de nouvelles sonorités, les chercheurs Garnet Hertz et Jussi Parika proposent le néologisme Zombie Media (2012). Ce terme fait référence aux objets techniques théoriquement dépassés, mais ramenés à la vie pour des usages plus ou moins éloignés de ceux pour lesquels ils ont été conçus. Le qualificatif « zombie » renvoie à un statut intermédiaire entre la fin de l'usage premier d'un objet et son réemploi pour d'autres usages. Il s'agit là d'une proposition féconde pour réfléchir à la postérité des objets numériques et à leur rôle éventuel en cas de crise sérieuse. Si le fait de devoir utiliser un PC 286 du début des années 1990 semble a priori absurde pour beaucoup de direction des systèmes d'information aujourd'hui, rappelons que c'est une situation courante dans des pays non occidentaux.

\section{Design web à basse consommation}

En dehors du hardware, les objectifs de réduction des émissions de carbone ont récemment joué un rôle important sur la production d'outils et de méthodes interrogeant la consommation énergétique du design numérique. Une première catégorie d'outils peut être identifiée comme moyens de mesurer, c'est-à-dire d'expliciter les émissions de carbone liées à la conception ou à l'usage de certains services et produits. Le relevé et l'explicitation des mesures se pratiquent malgré des problèmes méthodologiques et statistiques, comme la disparité du calcul de l'énergie nécessaire au transit d'un octet (Coroama et Hilty, 2014).

Ces outils de mesure ${ }^{7}$ correspondent à deux objectifs distincts : la prise de conscience des impacts par les designers et par les usagers. Dans le premier cas, Website Carbon fournit une empreinte carbone pour un trafic donné et des équivalences en kilogrammes de $\mathrm{CO}_{2}$. Le plug-in Carbonalyzer met en évidence, quant à lui, la consommation électrique et les émissions liées à un flux de données. Au-delà des impacts environnementaux donnés

7 https://www.websitecarbon.com 
Nova, N. \& Roussilhe, G. (2020). Du low-tech numérique aux numériques situés, Sciences du design, 11(1), p. 91-101.

(empreinte énergie/GES/eau), l'Ecoindex et la Web Energy Archive proposent un descriptif des éléments des pages, les éléments mis en cache, la complexité des pages invitant à modifier la conception et le développement des sites web.

Une dernière catégorie d'outils numériques vise la génération de sites prêts à l'emploi ou de solutions de gestion de contenus. C'est le cas du service txti ${ }^{8}$ qui permet de créer un blog très léger, du thème Wordpress qui ne pèse que 6 ko conçu par Jack Lenox ${ }^{9}$, ou encore du gestionnaire de contenus « léger » Cold $\mathrm{CMS}^{10}$.

Figure 4 : Interface de Carbonalyser

Si ces outils ne représentent pas une liste exhaustive de réponses aux enjeux de la crise environnementale, ils modifient les méthodes de conception et le périmètre d'action des designers numériques (Christie, 2013 ; Roussilhe, 2019). Un effet positif de ce design de la sobriété est l'accessibilité technique, car l'accès aux services numériques ainsi conçus s'ouvre à beaucoup de terminaux (vieux ordinateurs et téléphones intelligents) et s'adapte à une plus grande variété de réseaux de télécommunication $(2 \mathrm{G} / 3 \mathrm{G})$. Une autre

conséquence visible est la modification de l'approche graphique des outils numériques et de l'utilisation des médias (images/vidéos). Lors de sa refonte, le Low-tech Magazine a opté pour une utilisation intensive des couleurs, des caractères typographiques inclus de base dans les navigateurs, la disparition de leur logo, un traitement des images basé sur une réduction de leur poids ${ }^{11}$. Ce design web repose sur un triptyque de conception à faible impact (réduction du flux de données, diminution de la consommation énergétique, baisse des émissions de carbone) résumé dans la figure suivante ${ }^{12}$.

< insérer ici l'illustration suivante : image-05.jpg >

Figure 5 : Schéma du triptyque : réduction carbone / électricité / donnée et conséquences sur la conception

\section{Réseaux hétérogènes}

Ces réflexions à propos de la réduction de flux de données ne doivent cependant pas éclipser le fait qu'une telle problématique est déjà le lot quotidien de territoires en dehors du monde occidental. L'accès limité au réseau Internet filaire ou via les antennes de téléphonie mobile est une situation récurrente qui donne lieu à de multiples tactiques pour contourner cet écueil. Les projets Data Mules, MotoPost ou Daknet en Afrique et en Inde illustrent la possibilité de mise en place de systèmes de communication numérique à coût minime. Ceux-ci utilisent les moyens de transport conventionnels, voitures, motos et parfois animaux de traits, munis d'un ordinateur avec des capacités de stockage et un module de télécommunication limité (WiFi, Bluetooth) pour récupérer des messages et des contenus au gré du passage dans les villages, afin de faire circuler ensuite ceux-ci sur Internet une fois arrivé à un point de connexion dans une ville de taille plus importante. C'est ainsi que des courriels, des données produites par les médecins locaux, des fichiers

\footnotetext{
8 http://txti.es

9 https://sustywp.com

10 https://gitlab.com/hashbangfr/coldcms

$11 \mathrm{https} / / / w w w . l o w t e c h m a g a z i n e . c o m / 2018 / 09 /$ how-to-build-a-lowtech-website.html

12 Un tryptique qui a aussi une conséquente remarquable sur la question des données personnelles, puisque le fonctionnement du site web est prioritaire par rapport aux services tiers, cookies et autres services publicitaires collectant des données sur les utilisateurs, et qui sont du coup abandonnés.
} 
Nova, N. \& Roussilhe, G. (2020). Du low-tech numérique aux numériques situés, Sciences du design, 11(1), p. 91-101.

de tout ordre et parfois des demandes d'accès à des pages web sont stockés temporairement sur la machine du véhicule pour être transmis ensuite. Il s'agit autrement dit d'un réseau hétérogène et asynchrone, certes plus lent que les systèmes ordinaires, mais très utile dans de tels contextes. Dans un registre similaire, le cas d'el paquete à Cuba est éloquent. Ce terme fait référence à la livraison à domicile quasi institutionnalisée et hebdomadaire de contenus numériques téléchargés depuis Internet (films, images, musique, applications) par une personne qui apporte son disque dur à des particuliers. C'est là une forme d'accès à des contenus numérisés par déplacement physique des supports - tels que le DVD ou la clé USB - d'un ordinateur à un autre, plutôt que de transmettre les informations via un réseau informatique inexistant ou dont la bande passante serait trop faible (ou contrôlée). Fréquent dans les années 1980, ce mode d'échange est réinventé avec des disques durs, comme une sorte de cloud computing mobile dans des pays tels que Cuba.

Enfin, sans aller aussi loin, relevons le fait que les territoires occidentaux eux-mêmes ne sont pas toujours bien desservis par l'accès à Internet, ce qui donne lieu à la recherche de solutions pour pallier cet écueil. Dans ce cadre, la Fédération des fournisseurs d'accès Internet associatifs vise à décentraliser l'accès au réseau mondial. Ses membres fournissent un accès ADSL, ou construisent leur propre boucle locale, par exemple en couvrant de WiFi en point par point des zones mal desservies ${ }^{13}$.

\section{Création vidéoludique}

En écho au web design, les concepteurs de jeux vidéo de la scène indépendante s'attellent également à la création d'expériences ludiques reposant sur une forme d'économie de moyen, de minimalisme ou de réemploi potentiellement pertinent pour saisir ce à quoi une approche low-tech des contenus numériques pourrait ressembler. Sans entrer dans le thème de la réutilisation des briques logicielles, des jeux à l'esthétique visuelle, sonore et interactive minimale, tels que Return of the Obra Dinn, mais aussi les diverses expériences ludiques sur des consoles datées sont pertinents. Les compétitions de création sur C64 ou Amiga 500 dans un festival comme Datastorm, ou encore la publication de jeux expérimentaux tels que $A$ Slow Year sur Atari $2600^{14}{ }_{2}$ ou de cartouches musicales pour $\mathrm{C} 64^{15}$ en sont de bons exemples. Plus récemment, What Remains, produit pour la $\mathrm{NES}^{16}$, a justement pour but de combiner jeu d'aventure rétro et écologique sur une cartouche de console recyclée.

Figure 6 : DubCRT (Datadoor)

Il ne s'agit pas d'un design explicitement motivé par la crise environnementale, mais davantage de création numérique croisant une esthétique minimale avec une ingéniosité dans la programmation d'appareils à l'ergonomie et aux performances techniques très différentes. Là où certains voient dans ces exemples une forme de clin d'œil à une ère révolue des machines, soulignons d'une part que certains des porteurs de ces projets sont si jeunes qu'ils ne peuvent avoir de nostalgie pour des machines qu'ils n'ont pas connues, et d'autre part, que leur objectif est d'explorer des solutions à une création mainstream tournée vers la maximisation des performances graphiques. La démarche apparaît alors

\footnotetext{
13 https://ffdn.org/sites/default/files/field/docs/reponse-marche-fixe-ffdn.pdf

$14 \mathrm{http} / / /$ bogost.com/games/aslowyear/

15 https://datadoor.bandcamp.com/album/dubcrt

16 https://iodinedynamics.com/whatremains.html
} 
Nova, N. \& Roussilhe, G. (2020). Du low-tech numérique aux numériques situés, Sciences du design, 11(1), p. 91-101.

comme une voie valorisée socialement dans ces cercles permettant de se singulariser. En outre, ces projets témoignent du caractère non exclusivement fonctionnaliste et centré sur la résolution de problèmes d'un design low-tech, puisque des contenus à vocation plus conviviale comme le jeu vidéo peuvent en résulter.

\section{Des numériques situés}

Les cas que nous rapportons ici sont remarquables à plusieurs égards. D'abord, ils forment un corpus en cours de constitution, discuté par la communauté de designers comme sources d'inspiration ou solutions possibles à des manières de faire plus standards dans la profession. En cela, ils mettent en lumière des exemples à suivre (design de sites web à basse consommation), mais aussi des références pour penser autrement notre rapport aux objets techniques (esthétique minimale, hybridations de vieilles techniques à de nouvelles). Et ce, en particulier avec les cas cubains ou non occidentaux, en ne négligeant pas le fait que le low-tech possible envisagé par le monde occidental correspond aux situations vécues dans les pays du Sud. Par ailleurs, ils interrogent la notion même de «low-tech», terme souvent employé de manière générique sans définition très précise. Or ce qualificatif est en particulier problématique, puisqu'il implique une binarité avec le « high », alors que les cas cités dans cet article montrent l'existence de continuums multiples correspondant tantôt à l'énergie nécessaire à l'usage ou à la production, au réemploi plus ou moins important de matériaux recyclés ou de composants de périodes différentes, ou à des types de techniques diverses. De plus, les cas rapportés ici témoignent de toute une diversité de champs d'applications qui dépassent les motivations strictement utilitaristes : ils nous montrent que l'imaginaire de la sobriété numérique ne se cantonne pas à une absence de considérations symboliques ou esthétiques ${ }^{17}$.

Au vu des limites du terme « low-tech » employé dans le contexte des technologies de l'information et de la communication, nous proposons la notion de «numériques situés » qui décrirait plus précisément les phénomènes et les enjeux traversant la pratique du design dans le cadre de la crise environnementale au travers de trois dimensions ressortant des divers cas mentionnés dans cet article : (1) la nécessité de matérialisation des infrastructures et des impacts environnementaux, qui correspond à l'émergence des pratiques de design numérique liées à la mise en évidence des impacts écologiques du numérique, mais aussi à la récupération d'équipements, le réemploi ou la réappropriation de matériel électronique, par l'explicitation des conditions matérielles de fonctionnement (la jauge de batterie du Low-tech Magazine) ; (2) la question des territoires dans lesquels les infrastructures numériques sont déployées : leur territorialisation. Par exemple, la structure technique et la localisation du serveur du Low-tech Magazine à Barcelone le lie de fait aux conditions du territoire catalan (météo, ensoleillement). Et cette territorialisation invite à concevoir l'infrastructure technique et ses services à partir des spécificités du territoire, tout comme le el paquete cubain ou les boucles locales radio/fibre en France ; (3) la nécessité de la «terrestrialisation » : la réduction des impacts écologiques (GES, eutrophisation, etc.) visée par les exemples donnés inclut mais dépasse aussi le territoire. Par exemple, la diminution des émissions de carbone du numérique a pour but de contribuer à la stabilisation de la température moyenne sur Terre, bien au-delà de l'aire locale qu'elle pourrait concerner a priori. De même,

\footnotetext{
${ }^{17}$ Dans un autre registre, le travail de recherche de François Ribac sur la manière dont les arts de la scène réagissent aux problèmes posés par la cris environnementale, par le biais de modes de faire ou de réajustements sont aussi un exemple intéressant à consulter pour élargir le spectre des pratiques « low-tech» (Ribac, 2017).
} 
Nova, N. \& Roussilhe, G. (2020). Du low-tech numérique aux numériques situés, Sciences du design, 11(1), p. 91-101.

l'infrastructure numérique repose sur des réseaux globalisés : une réduction des impacts sera donc probablement distribuée à différents niveaux de ces réseaux planétaires ${ }^{18}$. Si cette notion de «numériques situés » propose comme logique de conception la matérialisation, la territorialisation et la terrestrialisation, elle n'en ouvre pas moins une dimension esthétique et symbolique forte, comme le démontrent les différents outils et contenus numériques observés ici.

< insérer ici l'illustration suivante : image-07.jpg >

Figure 7 : Composantes d'un numérique situé

\section{Conclusion}

Pour conclure sur cette note programmatique, soulignons que l'avènement de « numériques situés » impose l'évolution des pratiques des designers numériques. Si ceux-ci mobilisent ces exemples au sein de leurs sous-communautés diverses, il n'en reste pas moins que ces thématiques sont encore résolument minoritaires dans le design numérique. Cela pousse à repenser la formation de designers et l'évolution de leur implication en y intégrant : (1) des champs de connaissances techniques et sociales variés ; (2) la valeur des arbitrages liés aux enjeux de transition; et (3) de nouveaux champs de conception et de création numérique qui, au-delà de l'idéologie unique du progrès technique, pourraient créer un foisonnement d'approches originales intégrant les notions de territoire, d'énergie, d'empreinte carbone et/ou matérielle ou encore de plaisir.

\section{Bibliographie}

BIHOUIX, P. (2014). L'âge des low-tech : Vers une civilisation techniquement soutenable. Paris : Seuil.

CHRISTIE, J. (2013). Sustainable Web Design, A List Apart, [En ligne] https://alistapart.com/article/sustainable-web-design/

COROAMA, V. C. et HILTY, L. M. (2014). Assessing Internet energy intensity: A review of methods and results, Environmental Impact Assessment Review, 45, p. 63-68.

DIGUET, D. et LOPEZ, F. (2019). L'impact spatial et énergétique des data centers sur les territoires. Paris : Agence de l'Environnement et de la Maîtrise de l'Energie.

DORNE, G. (2019). Quel avenir pour les sites low-tech? Graphism. [En ligne] https://graphism.fr/quel-avenir-pour-les-sites-low-tech/

FING (2019). L'agenda pour un futur numérique et écologique. [En ligne] http://fing.org/agenda-futur-numerique-ecologique?lang=fr

GREENPEACE (2013). Emerging Trends in Electricity Consumption for Consumer ICT, Peter Corcoran and Andres Andrae in Greenpeace USA, Clicking Green.

\footnotetext{
18 Réduction de l'extraction des minerais en Afrique/Asie, des flux logistique moindre en Chine, gestion des déchets maîtrisée en local, ou stabilisation de la consommation énergétique du pays de production ou d'usage.
} 
Nova, N. \& Roussilhe, G. (2020). Du low-tech numérique aux numériques situés, Sciences du design, 11(1), p. 91-101.

HERTZ, G. et PARIKKA, J. (2012). Zombie Media: Circuit Bending Media Archaeology into an Art Method. Leonardo, 45(5), p. 424-430.

IRWIN, T. (2012). Transition Design: A Proposal for a New Area of Design Practice, Study, and Research, Design and Culture 7(2), p. 229-246.

NOVA, N. (2017). Démonter, extraire, combiner, remonter. Commodore 64 et créolisation technique, Techniques et Culture, 67-1, p. 116-133.

PITRON, G. (2018). La guerre des métaux rares. La face cachée de la transition énergétique et numérique. Uzès: Les Liens qui Libèrent.

RIBAC, F. (2017). Les récits de l'anthropocène. Quelle contribution des arts à la transition socio-écologique? Les Cahiers de l'atelier, Éditions de l'Atelier, p.103-108.

ROUSSILHE, G. (2019). Guide de conversion numérique au low tech. [En ligne] http://gauthierroussilhe.com/fr/posts/convert-low-tech

TOMLINSON, B., SIX SILBERMAN, M., PATTERSON, D., PAN, Y. et BLEVIS, E. (2012, 10 mai). Collapse informatics: augmenting the sustainability \& ICT4D discourse in HCI, Proceedings of the SIGCHI Conference on Human Factors in Computing Systems, Austin, Texas, USA, p. 655-664. 